\title{
Studies of eta-mesic nuclei at the LPI electron synchrotron
}

\author{
V.A. Baskov, A.V. Koltsov, A.I. L'vov, A.I. Lebedev, L.N. Pavlyuchenko, \\ V.V. Polyanskiy*, E.V. Rzhanov, S.S. Sidorin, G.A. Sokol \\ P.N. Lebedev Physical Institute, Leninsky prospect 53, Moscow 119991, Russia \\ Email: bolyapluton.lpi.troitsk.ru
}

\section{S.V. Afanasiev, A.I. Malakhov}

Joint Institute for Nuclear Research, Joliot-Curie 6, Dubna 141980, Moscow region, Russia

\section{A.S. Ignatov, V.G. Nedorezov}

Institute for Nuclear Research, 60-letiya Oktyabrya prospekt 7a, Moscow 117312, Russia

\begin{abstract}
A brief review of searches for $\eta$-mesic nuclei is presented with emphasis on photoreactions. Results of a new experiment done at the LPI electron synchrotron are reported. They are as follows.

New data on photoproduction of $\eta$-mesic nuclei off ${ }^{12} \mathrm{C}$ have been collected at the bremsstrahlung photon beam of $E_{\gamma \max }=850 \mathrm{MeV}$. An experimental setup with two plastic time-of flight spectrometers detected correlated $\pi^{+} n$ and $p n$ pairs from annihilation of $\eta$-mesons stopped in the nuclear matter and measured their velocity distributions. Data analysis was performed using an Intra Nuclear Cascade Model in the GEANT-3 framework in order to take into account properties of the setup and physical background. A separation between charged pions and protons was achieved using information on velocities and ranges of the particles in plastic detectors. The obtained data show, apart from previously observed $\pi^{+} n$ pairs from one-nucleon annihilation of etas (via $\eta N \rightarrow \pi N$ ), a presence of emitted correlated $p n$ pairs with velocities corresponding to the kinematics of the near-threshold reaction of two-nucleon absorption of the $\eta$-meson in the nucleus $(\eta N N \rightarrow N N)$. Assuming that such $\pi^{+} n$ and $p n$ pairs are mostly produced through formation and decay of quasi-bound states of the $\eta$-meson and a nucleus (i.e. $\eta$-mesic nuclei $\eta^{A}$ ), the cross section of $\eta$-mesic nuclei formation was estimated as $\sigma\left(\gamma+{ }^{12} \mathrm{C} \rightarrow \eta A+X\right) \lesssim 10 \mu \mathrm{b}$.
\end{abstract}

XXI International Baldin Seminar on High Energy Physics Problems,

September 10-15, 2012

JINR, Dubna, Russia

${ }^{*}$ Speaker. 


\section{Introduction: $\eta$-mesic nuclei}

$\eta$-mesic nuclei, i.e. nuclear systems $\eta A$ having the $\eta$-meson bound in a nuclear orbit by strong interaction with $A$ nucleons, have been predicted long ago [1, 2] - soon after recognizing the attractive character of the $\eta N$ interaction at low energies [3]. Observations and investigations of these exotic systems would be very valuable for understanding meson-baryon interactions in free space and in nuclei and for studies of properties of hadrons in the dense nuclear matter.

The $\eta$-meson, together with pions and kaons, belongs to the SU(3) octet of pseudoscalar mesons and has, therefore, a similar $q \bar{q}$ space structure. In contrast to the pion, however, the pseudoscalar coupling of $\eta$ to the nucleon is empirically rather small [П]. Nevertheless the amplitude of $\eta N s$ wave scattering is not as small as that for $\pi N$ scattering because of the contribution of the $s$-wave resonance $S_{11}(1535)$ which is actually a chiral partner of the nucleon - the lowest lying baryon with the opposite parity to the nucleon. This resonance has the mass slightly above the $\eta N$ threshold, $m_{\eta}+m_{N}=$ $1486 \mathrm{MeV}$, and owing to its very strong coupling to the $\eta N$ channel [with the branching

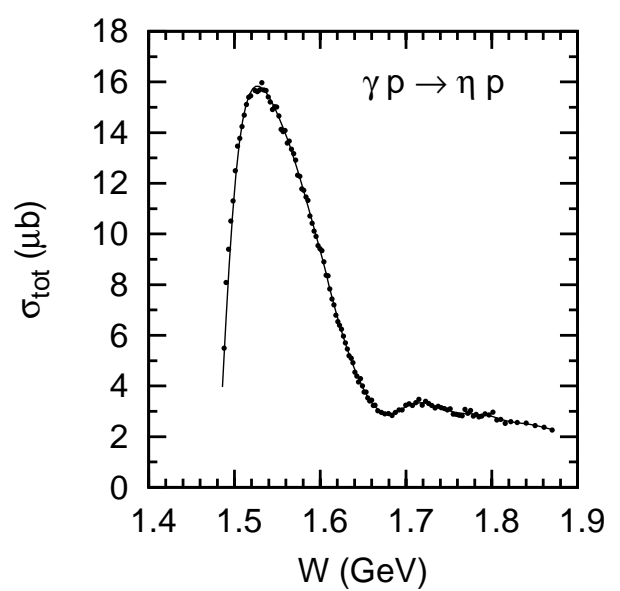

Figure 1: Total cross section of $\gamma p \rightarrow \eta p$ [5] as an illustration of the $S_{11}(1535)$ resonance strength in the $\eta N$ channel. ratio $\mathrm{Br}\left(S_{11}(1535) \rightarrow \eta N\right) \simeq 55 \%$ ] strongly enhances all interactions in this channel. A nice illustration of this feature is provided by Mainz data [5] on the total cross section of $\eta$ photoproduction off protons. A huge near-threshold enhancement shown in Fig. 1 is just a manifestation of the $S_{11}(1535)$ resonance excited in the reaction $\gamma p \rightarrow S_{11}(1535) \rightarrow \eta p$.

The $S_{11}(1535)$ resonance strongly contributes to the low-energy $\eta N$ scattering and, in particular, makes the threshold value of the $\eta N$ scattering amplitude (i.e. the $\eta N$ scattering length $a_{\eta N}$ ) positive. In the framework of a dynamical resonance model for the coupled channels $\pi N, \eta N$ and $\pi \pi N$, Bhalerao and Liu [阿] found

$$
a_{\eta N}=0.28+i 0.19 \mathrm{fm} \text {. }
$$

The positive value of $\operatorname{Re} a_{\eta N}$ means an effective attraction between $\eta$ and $N$, so that one can expect that several nucleons could jointly bind $\eta$ to a nuclear orbit. The first-order static-limit on-shell optical potential of $\eta$ in the nuclear matter at zero energy $E_{\eta}^{\mathrm{kin}}=0$ is equal to

$$
U(r)=-2 \pi a_{\eta N} \rho(r)\left(\frac{1}{m_{\eta}}+\frac{1}{m_{N}}\right),
$$

what gives [together with Eq. (11)] $U=-34-i 23 \mathrm{MeV}$ at normal nuclear matter density $\rho=\rho_{0}=$ $0.17 \mathrm{fm}^{-3}$. The imaginary part of the potential describes a local absorption rate $\Gamma=-2 \operatorname{Im} U$ of $\eta$ in the nuclear substance. 
With the above strength of the $\eta A$ potential, $\eta$-mesic nuclei $\eta A$ are expected to exist for all $A \geq$ 10 [6, 7]. Actually, due to a sharp (cusp) energy dependence of the $\eta N$ scattering amplitude near threshold, Fermi motion of nucleons and $\eta$ reduces the optical potential [especially its imaginary part], and this makes $\eta$-mesic nuclei to exist only for $A \geq 12$. For binding energies and widths of the lightest $\eta$-mesic nuclei Haider and Liu predicted [6, 元]

$$
\begin{aligned}
& E_{\eta}=-1.19 \mathrm{MeV}, \quad \Gamma_{\eta}=7.34 \mathrm{MeV} \text { for }{ }_{\eta}^{12} \mathrm{C}, \\
& E_{\eta}=-3.45 \mathrm{MeV}, \quad \Gamma_{\eta}=10.76 \mathrm{MeV} \text { for }{ }_{\eta}^{16} \mathrm{O}, \\
& E_{\eta}=-6.39 \mathrm{MeV}, \quad \Gamma_{\eta}=13.20 \mathrm{MeV} \text { for }{ }_{\eta}^{26} \mathrm{Mg} .
\end{aligned}
$$

Note, however, that a stronger $\eta N$ scattering amplitude was inferred in some other analyses. For example, using a $K$-matrix model for coupled channels $\pi N, \eta N, \gamma N$ and $\pi \pi N$, Green and Wycech [8, 9] found from fit to available data

$$
a_{\eta N}=(0.91 \pm 0.06)+i(0.27 \pm 0.02) \mathrm{fm} .
$$

With such a big strength of $\eta N$ interaction lighter $\eta$-mesic nuclei could also exist.

As an example of different predictions for binding energies and widths of $\eta$-mesic nuclei we mention very elaborated calculations [10-12], in which a model for meson-baryon interaction with dynamically generated resonances was build using a unitarized chiral perturbation theory for coupled channels $\pi N, \eta N, K \Lambda, K \Sigma$ and $\pi \pi N$ and then self-energies of all the particles in the nuclear matter were evaluated consistently. This approach leads to the $\eta N$ scattering length $a_{\eta N}=0.264+i 0.245 \mathrm{fm}$ close to that obtained in Eq. (1). The resulting $\eta A$ potential is, however, found stronger owing to nonlinear dressing effects: $U=-54-i 29 \mathrm{MeV}$ at normal nuclear density. Also stronger are $\eta$-meson bindings found in [12]:

$$
\begin{gathered}
E_{\eta}=-9.71 \mathrm{MeV}, \quad \Gamma_{\eta}=35.0 \mathrm{MeV} \text { for }{ }_{\eta}^{12} \mathrm{C} \\
E_{\eta}=-12.57 \mathrm{MeV}, \quad \Gamma_{\eta}=33.4 \mathrm{MeV} \text { for }{ }_{\eta}^{24} \mathrm{Mg}
\end{gathered}
$$

Bindings with equally large widths arise also in calculations [13 - 15] that use a chiral doublet model and treat $\eta A$ and $S_{11}(1535) A$ attraction as a result of partial restoration of chiral symmetry in the dense nuclear matter leading to reduction of the $S_{11}(1535)-N$ mass gap. It is clear that experimental data on energies and widths of $\eta$-mesic nuclei are needed to test these and many other models and calculations.

\section{Signature for eta-mesic nuclei produced in photoreactions}

A mechanism of $\eta$-mesic nuclei formation and decay in the photoreaction

$$
\gamma+A \rightarrow N^{\prime}+\eta(A-1) \rightarrow N^{\prime}+\pi+N+(A-2)
$$

is shown in Fig. Zna A fast nucleon $N^{\prime}$ ejected forward at the first stage of the reaction, i.e. in the subprocess

$$
\gamma+N^{\prime} \rightarrow N^{\prime}+\eta_{\text {slow }},
$$


escapes the nucleus, whereas a slow $\eta$ is captured by remaining $A-1$ nucleons to a bound state. At $E_{\gamma} \sim 800-900 \mathrm{MeV}$, a minimal momentum transfer to $\eta$ in the reaction (7) is not large (less than $70 \mathrm{MeV} / c$ ). That is why the total cross section of $\eta$-mesic nuclei formation off light nuclei (like carbon or oxygen implied in the following) turns out to be a few $\mu \mathrm{b}[16-21$, i.e. $\simeq 2-7 \%$ of the total cross section $\sigma_{\gamma A}^{\eta}$ of inclusive $\eta$ photoproduction, with the exact value strongly dependent on the assumed strength of the optical potential $U$.

a)

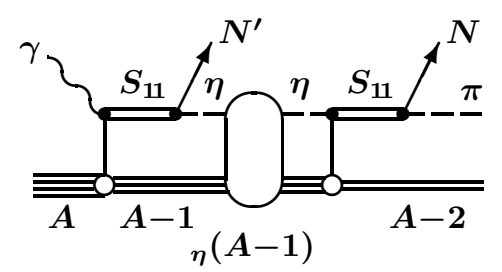

b)

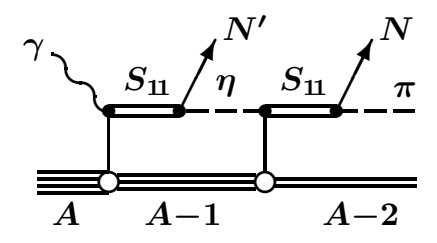

Figure 2: a) $\eta$-mesic nuclei formation and decay with the emission of back-to-back $\pi N$ pairs. b) Background creation of back-to-back $\pi N$ pairs by unbound $\eta$.

Energies $E[\eta(A-1)]$ of the produced $\eta$-mesic nuclei can, in principle, be determined through missing mass measurements in the reaction $(\gamma, p)$ using tagged photons $\gamma$ and a magnetic spectrometer for $N^{\prime}=p$. Indirectly, the same energy

$$
E[\eta(A-1)]=E_{\eta}+E_{A-1}=E_{\pi N}+E_{A-2}
$$

can also be found from the observed energy of a correlated back-to-back $\pi N$ pair produced at the second stage of the reaction (6) where the captured $\eta$ meson annihilates through the subprocess

$$
\eta N \rightarrow N \pi \text {. }
$$

The energy excitation of $(A-2)$ in $(8)$ is not a fixed value. It rather depends on whether an $s$-shell or $p$-shell nucleon $N$ is knocked out in the process (9). Therefore a distribution of the experimental observable $E_{\pi N}$ has appropriately a bigger width than the width of the $\eta$-mesic nucleus.

Neglecting binding and Fermi motion of nucleons and $\eta$, we have the following kinematical characteristics of the ejected correlated $\pi N$ pairs (as for energies, momenta and velocities):

$$
\begin{aligned}
& \sqrt{s}=E_{\pi}+E_{N}=m_{\eta}+m_{N}=1486 \mathrm{MeV}, \\
& E_{\pi}^{\text {kin }}=313 \mathrm{MeV}, \quad E_{N}^{\text {kin }}=94 \mathrm{MeV}, \quad p_{\pi}=p_{N}=431 \mathrm{MeV} / c \\
& \beta_{\pi}=0.95, \quad \beta_{N}=0.42 .
\end{aligned}
$$

A simple simulation that takes into account the Fermi motion of nucleons and $\eta$ as well as binding of these particles reveals that fluctuations around these ideal parameters are substantial (see Fig. 3) [specifically, we used in this simulation the $\eta$-meson binding energy of $10 \mathrm{MeV}$ with the width 25 $\mathrm{MeV}$; for nucleons, we assumed a Fermi-gas distribution with binding energies distributed between 5 and $30 \mathrm{MeV}$ ]. In particular, the angle $\theta_{\pi N}$ between the emitted pion and nucleon may not be so close to $180^{\circ}$, and a subtraction of background events with $\theta_{\pi N} \neq 180^{\circ}$ used sometimes in practice should be done cautiously. A shift of the peak down to $1486 \mathrm{MeV}$ in the distribution of the total energy $E_{\pi N}=E_{\pi}+E_{N}$ seen in Fig. 3 is related with binding of both the $\eta$-meson (by $10 \mathrm{MeV}$ ) and the nucleon (by $15 \mathrm{MeV}$ ). 

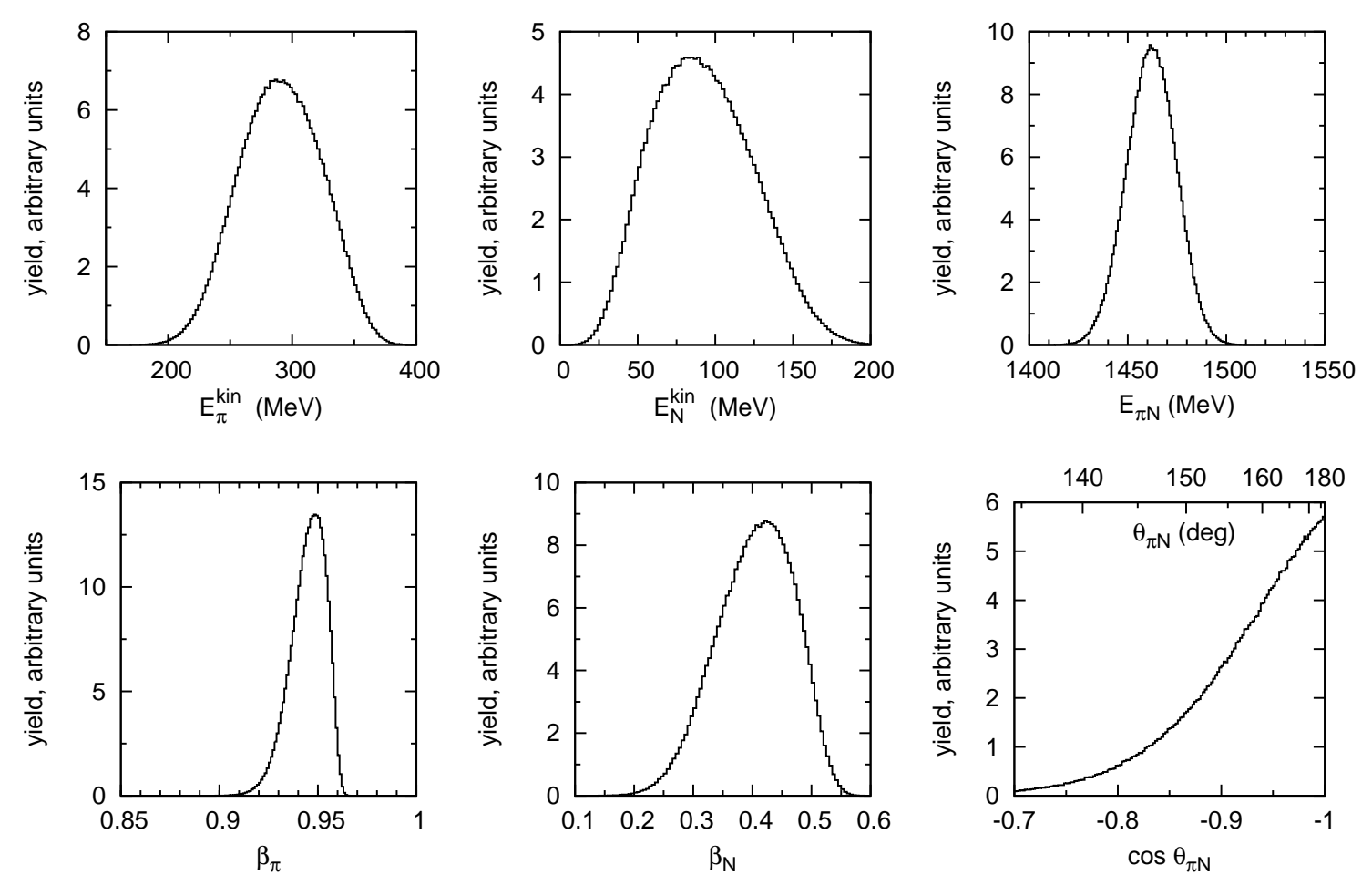

Figure 3: Simulation of $\pi N$ pairs emitted in $\eta$-mesic nuclei decays. Shown are distributions over kinetic energies of the particles, their total energy, velocities, and the $\pi N$ relative angle.

Notice that $\pi N$ pairs with the characteristics (10) do not necessary originate from $\eta$-mesic nuclei decays. They can also be produced by slow etas in a background nonresonance process shown in Fig. 2b. The resonance and nonresonance processes correspond to a resonance (Breit-Wigner) and nonresonance part of the full propagator [i.e. the Green function $\left.G\left(\boldsymbol{r}_{1}, \boldsymbol{r}_{2} ; E_{\eta}\right)\right]$ of the $\eta$-meson moving in the optical potential $U(r)$. Jointly, these parts generate a complicated spectrum of $E_{\eta}$ similar to that obtained in a toy model with a square-well potential [22, 23]. Shown in Fig. 4 is the spectral function in that model,

$$
S\left(E_{\eta}\right)=\iint \rho\left(\boldsymbol{r}_{1}\right) \rho\left(\boldsymbol{r}_{2}\right)\left|G\left(\boldsymbol{r}_{1}, \boldsymbol{r}_{2} ; E_{\eta}\right)\right|^{2} d \boldsymbol{r}_{1} d \boldsymbol{r}_{2},
$$

that characterizes near-threshold energy distribution of the propagated etas as well as the near-threshold energy dependence of the yield of $\pi N$ pairs produced by these $\eta$. Bound states of the $\eta$-meson give pronounced

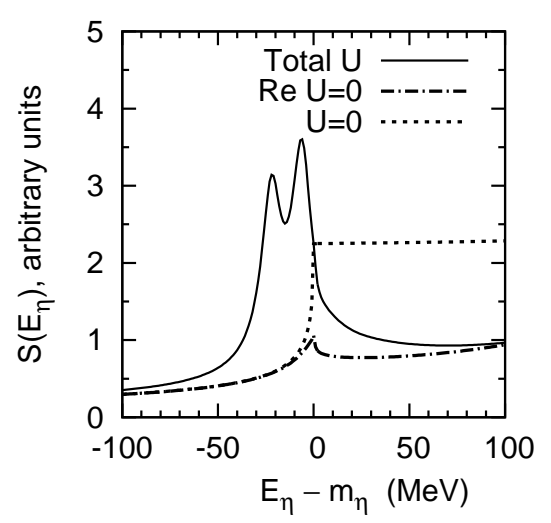

Figure 4: Spectral density of $E_{\eta}$ in a model with a rectangular-well attractive optical potential $U$ that approximately simulates the $\eta$-mesic nucleus ${ }_{\eta}^{12} \mathrm{C}$ [22]. The chosen potential was so strong that it bounded $\eta$ both in the $s$ and $p$-wave states. When the attractive potential is turned on, a pronounced peak (peaks) in the spectral density emerges at subthreshold resonance energy (energies). 
peaks in the yield of the $\pi N$ pairs at subthreshold energies $E_{\eta}$. Generally, observation of a relatively narrow resonance peak in the spectrum of $E_{\eta}$ in the region $E_{\eta}<m_{\eta}$ is mandatory for claiming an observation of $\eta$-mesic nuclei at all. We refer to recent works by Haider and Liu [24, 25] where a deeper and more elaborated consideration is given in relation with a recent experiment.

Since $\eta$ is isoscalar, the $\pi N$ pairs produced in the subprocess (9) have isospin $\frac{1}{2}$ and hence the following isotopic contents [for $\eta$-mesic nuclei with $A \gg 1$ ]:

$$
\operatorname{Br}(\pi N)=\left\{\begin{array}{l}
1 / 3 \text { for } \pi^{+} n \\
1 / 6 \text { for } \pi^{0} p \\
1 / 6 \text { for } \pi^{0} n \\
1 / 3 \text { for } \pi^{-} p
\end{array}\right.
$$

From these, the channel $\pi^{+} n$ was chosen for detection in our experiment.

\section{Previous searches for $\eta$-mesic nuclei}

Searches for $\eta$-mesic nuclei began very soon after their predictions [1] followed by suggestions [2, 16- 18, 26] to seek these novel high-energy nuclear excitations in missing-mass experiments using the inclusive reactions $\left(\pi^{+}, p\right)$ and $(\gamma, p)$.

The first two experiments have been done along this line in 1988 at Brookhaven [27] and Los Alamos [28, 29]. In both experiments, a $\pi^{+}$beam was used and several targets ( $\mathrm{Li}, \mathrm{C}, \mathrm{O}$ and $\mathrm{Al}$ ) were examined. The inclusive $\left(\pi^{+}, p\right)$ reaction

$$
\pi^{+}+A \rightarrow \eta(A-1)+p
$$

was studied in [27] with a magnetic spectrometer, whereas the Los Alamos experiment had also an additional $4 \pi$ BGO crystal ball for detecting charged paticles ejected in the subprocess (9) of $\eta$-mesic nuclei decays to $\pi N$ pairs in coincidence with the forward proton $p$.

The Brookhaven experiment did not find a theoretically expected signal [2] - a relatively narrow peak of a predicted strength in the missing mass spectrum. The team working at Los Alamos did report a preliminary evidence for a wanted peak for the ${ }^{16} \mathrm{O}$ target but this report was not confirmed (published) since then.

It was recognized in the following that the above obtained negative or incomplete results do not necessarily mean that the predicted $\eta$-mesic nuclei do not exist. It was possible that the binding energies and especially the widths of the $\eta$ bound states were theoretically underestimated. This point of view was supported by many-body calculations [30] taking into account some effects disregarded in the first theoretical works [1, 2], in particular — dressing, binding and collisional decays of the $S_{11}$ (1535) resonance in the dense nuclear matter. The analysis of [30] was later extended and revised [10-12] (in particular, dressing of mesons was also included) with the main conclusion survived that $\eta$-mesic nuclei widths are bigger than those found in [1], 包].

The next experiment has been performed at the Lebedev Physical Institute in Moscow/Troitsk [23, 31] (see also a summary in [32]). It was triggered [33, 34] by a suggestion [35] to seek $\eta$-mesic nuclei through observing decay products of $\eta$-mesic nuclei, namely two correlated back-to-back particles, a pion and a nucleon, ejected in the process of annihilation of captured $\eta$-mesons in the 
nucleus, Eq. (9). It was hoped that a background for the two very energetic particles (the pion and the nucleon) ejected in decays of $\eta$-mesic nuclei transversely to the beam would be lower than that for ejection of forward protons in the inclusive processes. Besides, it was hoped that background conditions in photon-induced reactions would be generally better than those in pion-induced ones.

Studies of the reaction

$$
\gamma+{ }^{12} \mathrm{C} \rightarrow\left({ }_{\eta}^{11} \mathrm{Be} \text { or }{ }_{\eta}^{11} \mathrm{C}\right)+N \rightarrow \pi^{+}+n+X+N
$$

done in the middle of 1990's at the LPI electron synchrotron indeed showed a signal of an enhanced production of the correlated back-to-back $\pi^{+} n$ pairs ejected transversely to the photon beam when the photon energy exceeded the $\eta$-meson photoproduction threshold. Energy resolution of the experimental setup was, however, not sufficient to resolve a peak similar to that shown in Fig. $⿴$ and to determine whether the observed correlated pairs were produced by bound or unbound intermediate etas.

After the works [23, 31] gaining and using information on the decay products became mandatory for experiment planning and data analysis in all further searches for $\eta$-mesic nuclei.

In 2004 an evidence for the $\eta$-mesic nucleus ${ }_{\eta}^{3} \mathrm{He}$ formed in the reaction

$$
\gamma+{ }^{3} \mathrm{He} \rightarrow{ }_{\eta}^{3} \mathrm{He} \rightarrow \pi^{0}+p+X
$$

has been reported from Mainz [36]. A resonance-like structure was observed in a contribution to the cross section from back-to-back $\pi^{0} p$ pairs found after a background subtraction. A later study [37] revealed, however, that the background has a rather complicated structure, so that the conclusions of Ref. [36] cannot be confirmed. At the moment their statement is that the existence of the $\eta$-mesic nucleus ${ }_{\eta}^{3} \mathrm{He}$ is not yet established.

One more attempt to find $\eta$-mesic nuclei by detecting their $\pi^{-} p$ decay products has recently been done at the JINR nuclotron [38]. The reaction studied was

$$
d+{ }^{12} \mathrm{C} \rightarrow\left({ }_{\eta}^{11} \mathrm{Be} \text { or }{ }_{\eta}^{11} \mathrm{C}\right)+N_{1}+N_{2} \rightarrow \pi^{-}+p+X+N_{1}+N_{2} .
$$

The measured effective mass spectra of the correlated back-to-back $\pi^{-} p$ pairs show a presence of resonance-like peaks lying slightly below the threshold energy $m_{\eta}+m_{N}=1486 \mathrm{MeV}$. However, a consistent interpretation of these peaks was not yet obtained.

To date the strongest evidence for the existence of $\eta$-mesic nuclei came from the precision COSY-GEM experiment [39]. Following ideas of the work [40] borrowed in turn from previous experience in studying deeply-bound pionic states in nuclei, the reaction

$$
p+{ }^{27} \mathrm{Al} \rightarrow{ }^{3} \mathrm{He}+{ }_{\eta}^{25} \mathrm{Mg} \rightarrow{ }^{3} \mathrm{He}+p+\pi^{-}+X
$$

of a recoilless formation of the $\eta$-mesic nuclei ${ }_{\eta}^{25} \mathrm{Mg}$ was explored and the mass of this $\eta$-mesic nucleus was determined through precision missing-mass measurements in $\left(p,{ }^{3} \mathrm{He}\right)$. A clear peak was found in the missing mass spectrum that corresponds to the binding energy $-13.13 \pm 1.64$ $\mathrm{MeV}$ and the width $10.22 \pm 2.98 \mathrm{MeV}$ of the formed $\eta$-mesic nucleus. An upper limit of $\approx 0.5 \mathrm{nb}$ was found for the cross section of the $\eta$-mesic nucleus formation.

Recently Haider and Liu argued [24, 25] that the observed peak in (17) is shifted down from the genuine binding energy of $\eta$ because of interference of the resonance and nonresonance mechanisms of the reaction (similar to those shown in Fig. 2). This very interesting effect signifies that the genuine $\eta$ binding in ${ }_{\eta}^{25} \mathrm{Mg}$ is $\approx-8 \mathrm{MeV}$ with the width $\approx 19 \mathrm{MeV}$. 


\section{On the two-nucleon decay mode of $\eta$-mesic nuclei}

The main novelty in our present research is exploring a new possibility for searching for $\eta$-mesic nuclei, namely through observation of their two-nucleon decay mode arising owing to the two-nucleon absorption of the captured $\eta$ in the nucleus,

$$
\eta N N \rightarrow N N
$$

see Fig. 5. Ejected in this process correlated back-toback nucleons of the $N N$ pairs have very high energies $\left(E_{N}^{\mathrm{kin}} \simeq \frac{1}{2} m_{\eta}=274 \mathrm{MeV}\right)$ and momenta $\left(p_{N} \simeq\right.$ $770 \mathrm{MeV} / c$ ), so that they are to be visible (especially in coincidence) at the background of other particles emitted in photoreactions at $E_{\gamma} \sim 800 \mathrm{MeV}$ and thus should provide a bright signature for the $\eta$-mesic nucleus formation.

The $N N$ pair production in decays of $\eta$ in the nuclear matter was considered among other channels by Chiang, Oset and Liu [30] in terms of the selfenergy of $S_{11}(1535)$ that includes a contribution of $S_{11}(1535) N \rightarrow N N$. A more direct and rather transparent evaluation of this process has been done by Kulpa and Wycech [41] who used available experimental data on the inverse reactions $p p \rightarrow p p \eta, p n \rightarrow p n \eta$ and $p n \rightarrow d \eta$ and then converted them into the rate of (18). In terms of the imaginary part $W_{N N}$ of the optical potential $U$, this rate was found to be proportional to $\rho^{2}$, being $W_{N N}=3.4 \mathrm{MeV}$ at central nuclear density. This is only about $15 \%$ of $W_{N} \sim 23 \mathrm{MeV}$ related with the absorption of $\eta$ by one nucleon. Nevertheless such a small fraction of $N N$ can be quite visible experimentally because of a specific isotopic contents of the $\pi N$ and $N N$ pairs.

The matter is that $\gtrsim 90 \%$ of these $N N$ pairs are proton plus neutron because the cross section of $p p \rightarrow p p \eta$ (and $n n \rightarrow n n \eta$ ) is by order or magnitude less than that of $p n \rightarrow p n \eta$ (plus $p n \rightarrow d \eta$ ), see Fig. 6 where pertinent Uppsala-Celsius [42-45] and COSY [46, 47] data are shown (and see also, e.g., [48] for theoretical explanations). This difference can be traced to isospin factors and Fermi statistics signs in the dominating pion-exchange mechanism of the reaction $N N \rightarrow N N \eta$ shown in Fig. 7. If the experimental setup detects one charged and one neutral particle from the pairs, it detects $\sim 90 \%$ of $N N$ and only $\sim 33 \%$ of $\pi N$. Then count rates of the setup would not be so different for $p n$ and $\pi^{+} n$ pairs. That seems to be exactly what we see in our experiment.

Neglecting binding effects and effects of Fermi motion of nucleons and $\eta$, we have the following kinematical characteristics of the correlated $N N$ pairs (i.e. energies, momenta, velocities) 

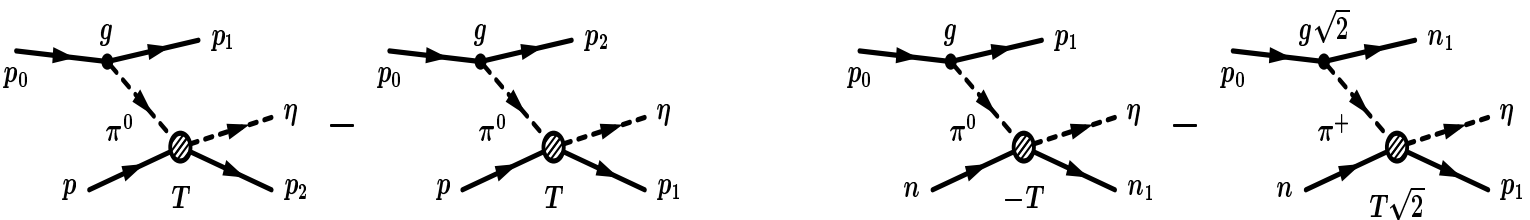

Figure 7: Pion-exchange mechanism of $N N \rightarrow N N \eta$. Isospin factors, which accompany the $\pi N N$ coupling $g$ and the $\pi N \rightarrow \eta N$ amplitude $T$, and the Fermi-statistics signs (both shown in this Figure) jointly determine the big difference between the cross sections of $p p \rightarrow p p \eta$ and $p n \rightarrow p n \eta$ (plus $p n \rightarrow d \eta$ ). Antisymmetrization of the initial state and initial/final state interactions are not shown.
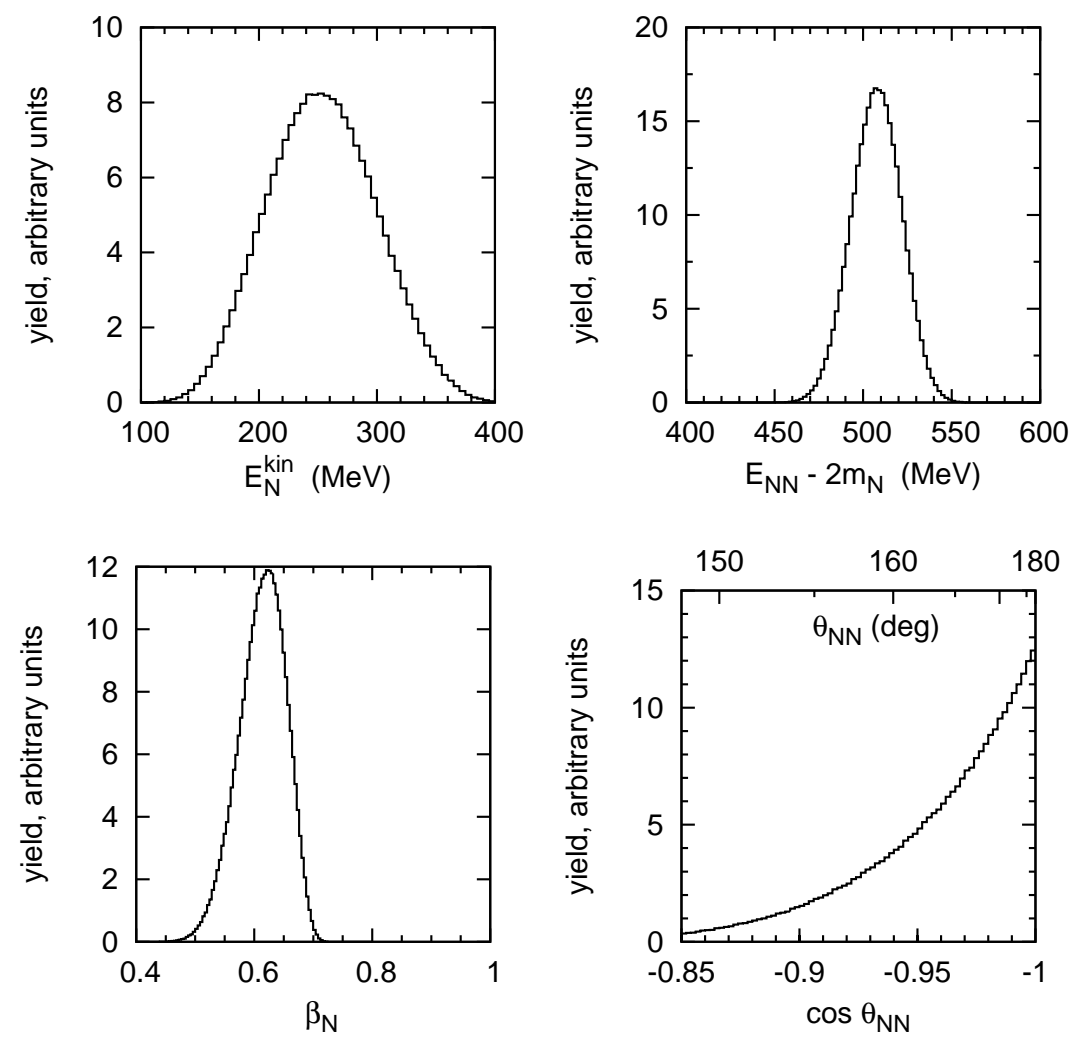

Figure 8: Simulation of $N N$ pairs emitted in decays of $\eta$-mesic nuclei. Shown are distributions over the kinetic energy and velocity of one of the nucleons, the total energy of the pair and the relative angle.

ejected in $\eta$-mesic nuclei decays:

$$
E_{N_{1}}^{\mathrm{kin}}=E_{N_{2}}^{\mathrm{kin}}=\frac{1}{2} m_{\eta}=274 \mathrm{MeV}, \quad p_{N_{1}}=p_{N_{2}}=767 \mathrm{MeV} / c, \quad \beta_{N_{1}}=\beta_{N_{2}}=0.63 .
$$

Actually, the Fermi motion and binding leads to fluctuations around these ideal parameters as a simple simulation reveals, see Fig. 8. Note that the angular correlation in the $N N$ pairs is stronger than that in the $\pi N$ pairs - owing to higher momenta of particles in $N N$. 
The first studies of the photoreaction

$$
\gamma+{ }^{12} \mathrm{C} \rightarrow\left({ }_{\eta}^{11} \mathrm{Be} \text { or }{ }_{\eta}^{11} \mathrm{C}\right)+N \rightarrow p+n+X+N
$$

have recently been done at the LPI synchrotron and we report below on the obtained results.

\section{Experimental setup at LPI}

Our experiment was performed at the bremsstrahlung photon beam of the 1.2-GeV electron synchrotron of the Lebedev Physical Institute. Photons were produced with an electron beam of intensity $I_{e} \simeq 10^{12} \mathrm{~s}^{-1}$ and the duty factor $\simeq 10 \%$. The energy of the beam was usually $E_{e}=E_{\gamma \max }=850 \mathrm{MeV}$ (i.e. above the $\eta$ photoproduction threshold off free nucleons, $E_{\eta}$ thr $=708 \mathrm{MeV}$ ); additional measurements of subthreshold backgrounds have been done at $E_{e}=E_{\gamma \max }=650 \mathrm{MeV}$.

The experimental setup included two time-of-flight arms (two scintillation telescopes - $\mathrm{C}$ and $\mathrm{N}$ arms) for detecting in coincidence charged and neutral particles (back-to-back pairs), see Fig. 9. These arms were both positioned at $90^{\circ}-90^{\circ}$ with respect to the beam axis in order to minimize background.

The $\mathrm{C}$-arm used for detection of charged particles is a plastic TOF spectrometer for charged pions and protons. It consists of a start detector T1 $\left(20 \times 20 \times 2 \mathrm{~cm}^{3}\right)$, a stop detector T2 $(50 \times$ $\left.50 \times 5 \mathrm{~cm}^{3}\right)$ and three energy losses detectors $\Delta E_{1}, \Delta E_{2}$ and $\Delta E_{3}$

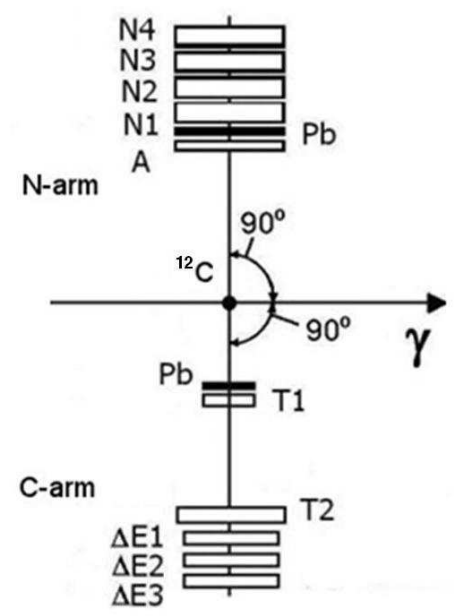

Figure 9: Layout of the experimental setup at LPI. $\left(40 \times 40 \times 2 \mathrm{~cm}^{3}\right.$ each). A $4 \mathrm{~mm}$ lead $(\mathrm{Pb})$ plate was used in some runs for TOF calibrations with ultrarelativistic electrons/positrons produced in the lead plate with high-energy photons emitted from the target owing to production and decays of neutral pions.

The $\mathrm{N}$-arm is a plastic TOF spectrometer for neutrons. It consists of a veto counter A $(50 \times$ $\left.50 \times 2 \mathrm{~cm}^{3}\right)$ and four plastic blocks - the neutron detectors N1, N2, N3 and N4 $\left(50 \times 50 \times 10 \mathrm{~cm}^{3}\right.$ each). Again, a $4 \mathrm{~mm}$ lead plate was used in some runs for TOF calibrations. The efficiency of the $\mathrm{N}$-arm for neutrons of energies above $50 \mathrm{MeV}$ was $\approx 30 \%$.

In both arms each volume of scintillator counters/blocks was viewed from corners with 4 phototubes. The time-of-flight bases in the $\mathrm{C}$ and $\mathrm{N}$ arms were $1.4 \mathrm{~m}$ and the time resolution was $\simeq 200 \mathrm{ps}(1 \sigma)$. The target was a carbon cylinder of the $10 \mathrm{~cm}$ length along the beam axis. Its diameter was $4 \mathrm{~cm}$, i.e. slightly more than the diameter of the collimated photon beam $(3 \mathrm{~cm})$. The distance between the target and the start detector T1 was $0.7 \mathrm{~m}$.

Mostly, the setup was the same as in our previous work [31, 32] but a few useful changes have been made:

- $\Delta E_{i}$ detectors have been placed after the time-of-flight interval T1-T2. This enabled us to have a better $\pi^{ \pm} / p$ separation and time resolution.

- A transverse size of the start detector T1 was cut off according to required geometry. This reduced a background load of the $\mathrm{C}$-arm. 
- A thickness of the start detector was also reduced in order to improve time resolution.

- All unnecessary layers of absorbers used previously to suppress radiative backgrounds have been removed from the time-of-flight interval, with the effect of reducing the $e^{+} / e^{-}$background created by photons from $\pi^{0}$ decays.

General tests of the setup, including preliminary time calibrations of the arms, have been done in special runs, in which the quasifree reaction $\gamma p \rightarrow \pi^{+} n$ inside carbon nuclei was observed. In such runs the two arms of the setup have been moved to the angles $50^{\circ}-50^{\circ}$ where the high count rate enabled one to do the calibrations quickly. Lead convertors used in these runs provided reliable ultrarelativistic reference points $\beta=1$ for particle's velocities $\beta_{C}$ and $\beta_{N}$ measured in the $\mathrm{C}$ - and $\mathrm{N}$-arms. A two-dimensional $\beta_{C}-\beta_{N}$ plot on Fig. 10 illustrates this procedure.

The calibration done provided a linear scale of velocities in the range $\beta=0.6-1$ with errors of about $3 \%$ $(1 \sigma)$. We have checked the linearity of the scale by using cosmic rays and setting different distances between detectors.

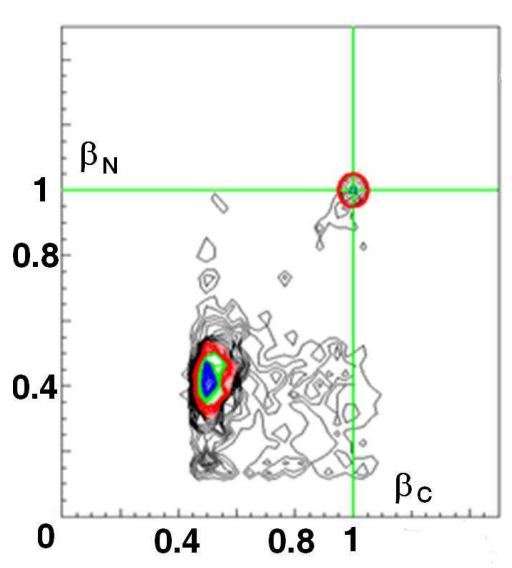

Figure 10: A two-dimensional plot of particle velocities, $\beta_{C}$ and $\beta_{N}$, in the $\mathrm{C}$ and $\mathrm{N}$ arms.

\section{Results and comparison with simulations}

Measurement runs have mostly been done in 2009 at two maximal beam energies: $E_{\gamma \max }=$ $650 \mathrm{MeV}$ and $850 \mathrm{MeV}$. The on-line trigger was a coincidence of particles in the C-and $\mathrm{N}$-arms within a time gate of $50 \mathrm{~ns}$.

For further off-line analysis events were selected with an additional condition of sufficiently long ranges of the charged particles,

$$
\Delta E_{i}>E_{i}^{\mathrm{thr}} \text { for all } i=1,2,3
$$

with experimentally adjusted thresholds $E_{i}^{\text {thr }}$. In this way low-energy particles in the C-arm were rejected.

A two-dimensional histogram in the variables $\Delta E-\beta_{C}$, where $\Delta E$ is the minimal energy loss in the $\Delta E_{i}$ detectors,

$$
\Delta E=\min _{i}\left(\Delta E_{i}\right)
$$

is shown in Fig. 11 for the beam energy $E_{\gamma \max }=850 \mathrm{MeV}$. Results of simulations using the Intra Nuclear Cascade (INC) model [49] in the GEANT-3 framework are shown in Fig. 12 for comparison. The INC model takes into account production of various mesons and baryon resonances, their free propagation in the nuclear matter, and then various $2 \rightarrow 2$ collisional reactions including $\eta N \rightarrow \pi N$. This model successfully describes many photoreactions in wide kinematical ranges as was demonstrated, beyond [49], in simulations of the GRAAL experiment at energies 500-1500 
$\mathrm{MeV}$ [50]. Binding effects for $\eta$ and reactions like $\eta N N \rightarrow N N$ were not included into the model, so one can try to find effects arising due to formation and decay of $\eta$-mesic nuclei through characteristic deviations of the model predictions from the experimental data.

The simulation shows that the selection (21) of particles with sufficiently long ranges distinguishes very well protons (as particles with $\beta_{C} \leq 0.7$ ) and pions (as particles with $\beta_{C} \geq 0.7$ ): the overlap is less than $1 \%$.

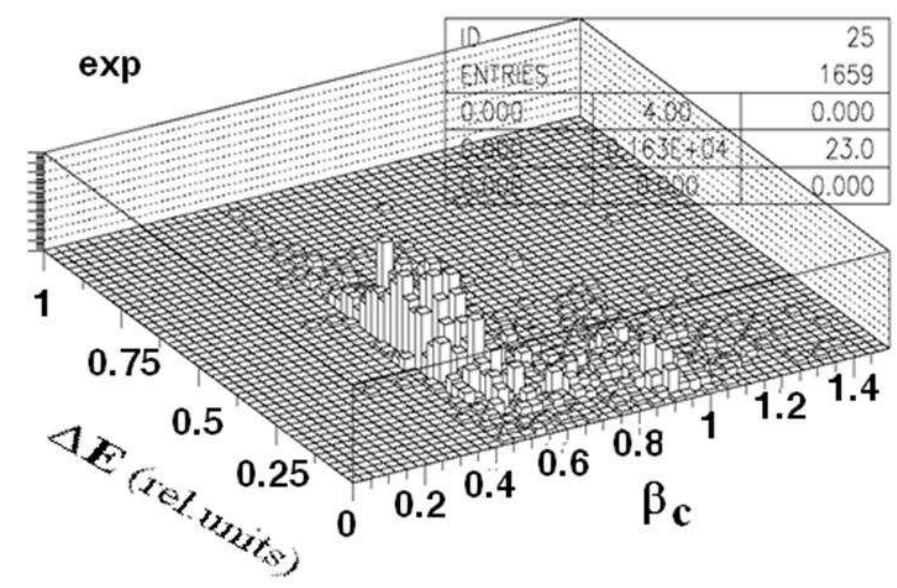

Figure 11: Two-dimensional $\Delta E-\beta_{C}$ distribution, experiment.

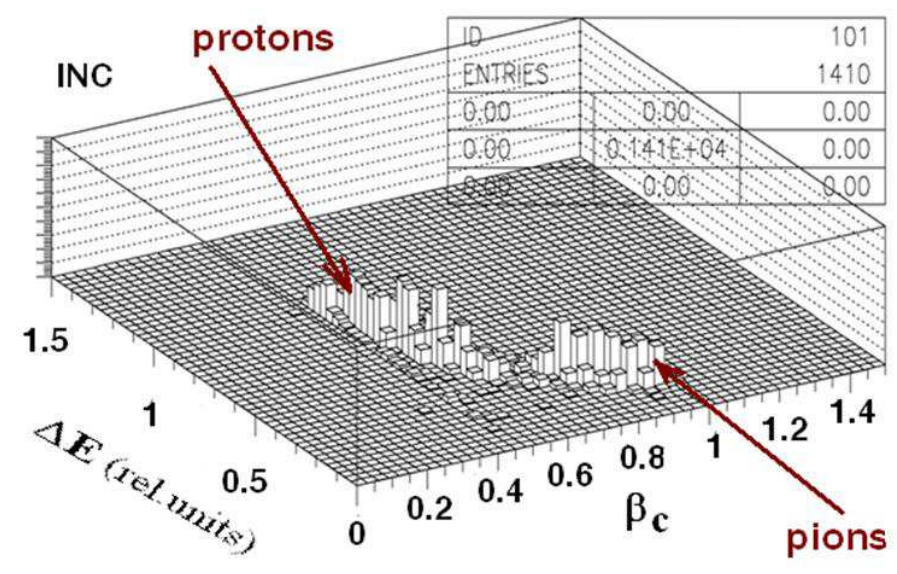

Figure 12: Two-dimensional $\Delta E-\beta_{C}$ distribution, the INC model.

Considering one-dimensional spectra over $\beta_{C}$ of events selected according to the condition (21) of sufficiently long ranges and imposing the additional cut-off $0.3<\beta_{N}<0.7$ for neutron velocities, we find rather interesting structures in the spectra. Shown in Fig. 13 are experimental data (blue areas) together with results of the INC simulation (pink hatched areas). Separately shown are INC predictions for the number of protons and charged pions in the C-arm. There is a qualitative agreement of the INC simulation with the experimental data for the case of the 
subthreshold beam energy, $E_{e}=650 \mathrm{MeV}$. Meanwhile, in the case of $E_{e}=850 \mathrm{MeV}$ there is a clear excess of the experimentally observed events over the simulation results in two velocity regions closely corresponding to the kinematics of $\eta$-mesic nuclei decays with emission of $\pi N$ and $N N$ correlated pairs, Eqs. (10) and (19).

Knowing from the INC simulations that the "normal" (without $\eta$-mesic nuclei) dynamics of the considered reaction does not yield a sufficient amount of protons and pions with the velocities of about $\beta_{C} \sim 0.7$, we interpret the found anomaly at $\beta_{C} \sim 0.7$ as a result of production of low-energy $\eta$-mesons followed by their two-nucleon annihilation.

The energy resolution of the experimental setup is not sufficient to say whether an essential part of these $\eta$-mesons is produced in the bound state, but theoretical arguments discussed in above make such a statement plausible.

Concerning the excess of pions with $\beta_{C} \simeq 0.95$, this feature is in agreement with our measurements reported earlier [23, 31, 32]. It can be interpreted as an evidence for one-nucleon annihilation of produced low-energy $\eta$-mesons (bound or unbound).

Electron/positron peaks shown in Fig. 13 originate from calibration runs with the lead plate inserted. They were not included into simulations made.
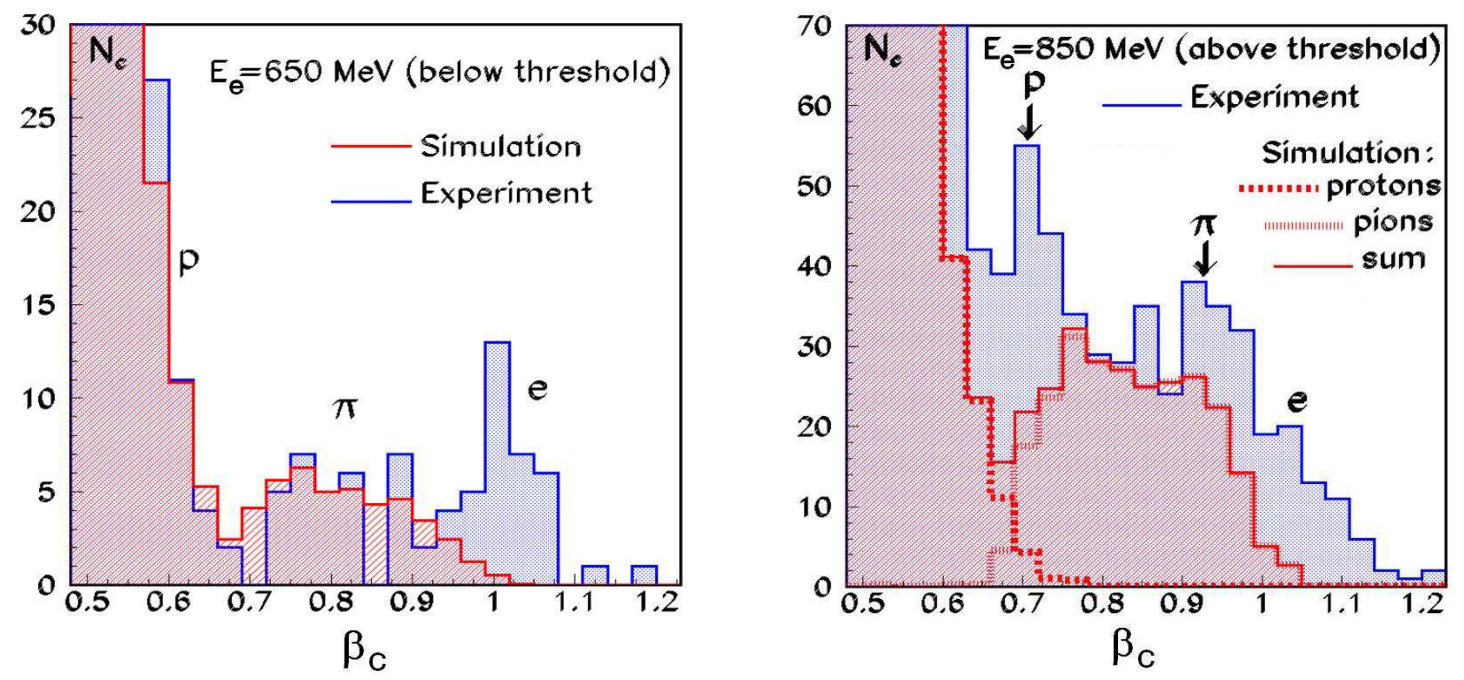

Figure 13: Velocity distribution of charged particles selected according to the criterion $\Delta E_{i}>0$ (for all $i=1,2,3)$ at $E_{e}=650$ and $850 \mathrm{MeV}$. A well visible excess of events over the INC simulation is seen at the right panel — in the case of the beam energy exceeding the $\eta$-photoproduction threshold — in both velocity regions corresponding to the expected velocities of the $\pi N$ and $N N$ decay products of $\eta$-mesic nuclei.

The observed proton peak in the $\beta_{C}$ distribution is very unusual because it corresponds to $p n$ pairs with very high kinetic energies $T_{p} \sim T_{n} \sim 200-300 \mathrm{MeV}$ and transverse momenta $p_{p} \sim$ $p_{n} \sim 400-800 \mathrm{MeV} / \mathrm{c}$. One should keep in mind that photons which produce such pairs have quite a modest energy $650 \mathrm{MeV}<E_{\gamma}<850 \mathrm{MeV}$. Ordinary photoproduction reactions do not give nucleons with such a high energy and momentum. Creation and annihilation of intermediate low-energy $\eta$-mesons seems to be the only explanation to these events. 
Assuming that the observed access events are mainly related with formation and isotropic decays of $\eta$-mesic nuclei with $A=11$, we can estimate their photoproduction cross section. The number of photons of the energies $E_{\gamma}=650-850 \mathrm{MeV}$ that hit the carbon target in experimental runs was evaluated via comparison of the total yield of charged pions detected by a single $\mathrm{C}$-arm of the setup with predictions of INC for that yield, thus giving the result $N_{\gamma} \simeq 1.36 \times 10^{11}$. Taking into account the solid angle of the C-arm telescope $\left(\Omega_{C}=0.027 \mathrm{sr}\right)$, efficiencies of detectors, a geometric efficiency of the $N$-arm of the setup $(\sim 18 \%$ as found from theoretically expected angular distributions of particles of the correlated pairs), we arrived at the following cross section of $\eta$-mesic nucleus formation:

$$
\sigma\left(\gamma+{ }^{12} \mathrm{C} \rightarrow{ }_{\eta} A+X\right) \lesssim 10 \mu \mathrm{b} .
$$

We write it as an upper limit because part of the observed events can be related with unbound etas. This number is consistent with available theoretical estimates (typically, a few $\mu \mathrm{b}$ ).

\section{Conclusions}

The new obtained data confirm the main features of the $\pi N$ signal of formation and decay of $\eta$-mesic nuclei off the carbon target in the photoreaction found in our previous work.

A new signature for formation and decay of $\eta$-mesic nuclei, the back-to-back $p n$ pairs, was explored. For the first time an experimental evidence was found that the yield of such pairs in the region of $\beta_{C} \sim 0.6-0.7$ is quite large and therefore is also suitable for searching for $\eta$-mesic nuclei.

Assuming that the observed excess of events is related with $\eta$-mesic nuclei, an estimate of the total cross section of formation of $\eta$-nuclei in the photoreaction off carbon have been obtained, see Eq. (23).

We have plans to carry out a more precise experiment, with a better energy resolution, at the deuteron beam of the JINR nuclotron.

\section{Acknowledgments}

This work was supported in part by the RFBR grants 08-02-00648-a and 10-02-01433-a. A nice work of the accelerator group of the LPI synchrotron and its leader G.G. Subbotin is highly appreciated.

\section{References}

[1] Q. Haider and L.C. Liu, Formation of an eta-mesic nucleus, Phys. Lett. B 172 (1986) 257; Erratum: Ibid. 174 (1986) 465E.

[2] L.C. Liu and Q. Haider, Signature for the existence of eta-mesic nuclei, Phys. Rev. C 34 (1986) 1845.

[3] R.S. Bhalerao and L.C. Liu, Off-shell model for threshold pionic $\eta$ production on a nucleon and for $\eta N$ scattering, Phys. Rev. Lett. 54 (1985) 865.

[4] L. Tiator, C. Bennhold and S.S. Kamalov, The $\eta N N$ coupling in eta photoproduction, Nucl. Phys. A $\mathbf{5 8 0}$ (1994) 455. 
[5] E.F. McNicoll, S. Prakhov, I.I. Strakovsky et al., Experimental study of the $\gamma p \rightarrow \eta p$ reaction with the Crystal Ball detector at the Mainz Microtron (MAMI-C) [data tables are available from the Durham HEPDATA base, http: / /durpdg.dur.ac.uk].

[6] Q. Haider and L.C. Liu, Dependence of calculated binding energies and widths of $\eta$-mesic nuclei on treatment of subthreshold $\eta$-nucleon interaction, Phys. Rev. C 66 (2002) 045208.

[7] Q. Haider and L.C. Liu, Eta-mesic nucleus: A new form of nuclear matter, Acta Phys. Pol. B Proc. Suppl. 2 (2009) 121.

[8] A.M. Green and S. Wycech, $\eta$-nucleon scattering length and effective range, Phys. Rev. C 55 (1997) R2167.

[9] A.M. Green and S. Wycech, $\eta$-nucleon scattering length and effective range uncertainties, Phys. Rev. C 71 (2005) 014001.

[10] T. Inoue, E. Oset and M.J. Vicente Vacas, Chiral unitary approach to S-wave meson baryon scattering in the strangeness $S=0$ sector, Phys. Rev. C 65 (2002) 035204.

[11] I. Inoue and E. Oset, $\eta$ in the nuclear medium within a chiral unitary approach, Nucl. Phys. A 710 (2002) 354.

[12] C. García-Recio, T. Inoue, J. Nieves and E. Oset, $\eta$ bound states in nuclei, Phys. Lett. B 550 (2002) 47.

[13] D. Jido, H. Nagahiro and S. Hirenzaki, Medium effects to the $N(1535)$ resonance and $\eta$ mesic nuclei, Phys. Rev. C 66 (2002) 045202.

[14] H. Nagahiro, D. Jido and S. Hirenzaki, Formation of mesic nuclei by $(\gamma, p)$ reactions, Nucl. Phys. A 761 (2005) 92.

[15] D. Jido, E.E. Kolomeitsev, H. Nagahiro and S. Hirenzaki, Level crossing of particle-hole and mesonic modes in eta-mesonic nuclei, Nucl. Phys. A 811 (2008) 158.

[16] M. Kohno and H. Tanabe, Low energy $\eta$ production in $\left(\pi^{+}, p\right)$ and $(\gamma, p)$ reactions on ${ }^{12} C$, Phys. Lett. B 231 (1989) 219.

[17] A.I. Lebedev and V.A. Tryasuchev, Calcultion of the photoptoduction cross section of $\eta$-nuclei, Voprosy Atomnoi Nauki i Tekhniki, ser. Yad.-Fiz. Issled. (Kharkov), 8/8 (1989) 97 (in Russian).

[18] A.I. Lebedev and V.A. Tryasuchev, Cross section for production of $\eta$ nuclei by photons, J. Phys. G: Nucl. Part. Phys. 17 (1991) 1197.

[19] A.I. Lebedev and V.A. Tryasuchev, Study of the photoproduction of eta mesic nuclei on the basis of a complex potential, Phys. Atom. Nucl. 58 (1995) 586 [Yad. Fiz. 58 (1995) 642 (in Russian)].

[20] V.A. Tryasuchev, Photoproduction of light eta nuclei, Phys. Part. Nucl. 30 (1999) 606 [Fiz. Elem. Chast. Atom. Yadra 30 (1999) 1391 (in Russian)].

[21] V.A. Tryasuchev, Theoretical analysis of the formation of $\eta$ mesic nuclei in $\gamma+A \rightarrow N+{ }_{\eta} A^{\prime}$ reactions, Phys. Atomic Nucl. 64 (2001) 346 [Yad. Fiz. 64 (2001) 396 (in Russian)].

[22] A.I. L'vov, Production and decay of eta-mesic nuclei, in Proc. of the 7th Int. Conf. 'Mesons and Light Nuclei', Czech Republic, 1998 (Mesons and Light Nuclei '98, World Scientific, Eds. J. Adam, P. Bydžovský, J. Dobeš, R. Mach, J. Mareš and M. Sotona), pp. 469-472; E-print arXiv: nucl-th/9809054.

[23] G.A. Sokol, T.A. Aibergenov, A.V. Kravtsov, A.I. L'vov and L.N. Pavlyuchenko, Search for $\eta-m e s i c$ nuclei in photoproduction processes, Fizika B 8 (1999) 85. 
[24] Q. Haider and Lon-Chang Liu, Eta-mesic nucleus and COSY-GEM data, Acta Phys.Polon. B 41 (2010) 2231.

[25] Q. Haider and Lon-Chang Liu, Interference and nuclear medium effects on the eta-mesic nuclear spectrum, J. Phys. G: Nucl. Part. Phys. 37 (2010) 125104.

[26] M. Kohno and H. Tanabe, Pion-induced $\eta$ production on nuclei, Nucl. Phys. A 519 (1990) 755.

[27] R.E. Chrien, S. Bart, P. Pile et al., Search for bound states of the $\eta$ meson in light nuclei, Phys. Rev. Lett. 60 (1988) 2595.

[28] B.J. Lieb, in Proceedings of International Conference on Nuclear Physics, Sao Paulo, Brazil, 1988.

[29] B.J. Lieb, L.C. Liu, E. Cheung et al., Search for nuclear bound states of the eta meson, Progress at LAMPF, January - December 1988. LA-11670-PR Progress Report, pp. 52-55.

[30] H.C. Chiang, E. Oset and L.C. Liu, Width of bound eta in nuclei, Phys. Rev. C 44 (1991) 738.

[31] G.A. Sokol, T.A. Aibergenov, A.V. Koltsov et al., Discovery of $\eta$-mesic nuclei, Part. Nucl. Lett. 102 (2000) 71 [Pisma EChaYa No.5 [102] (2000) 71 (in Russian)].

[32] G.A. Sokol and L.N. Pavlyuchenko, Discovery and investigation of $\eta$-mesic nuclei in photoproduction processes, Phys. At. Nucl. 71 (2008) 509 [Yad. Fiz. 71 (2008) 532 (in Russian)].

[33] G.A. Sokol, V.L. Kashevarov, A.I. Lebedev and L.N. Pavlyuchenko, Photoproduction of eta-nuclei, in Proceedings of International Conference on Mesons and Nuclei at Intermediate Energies, Dubna, Russia, 1994 (Eds. M.Kh. Khankhasayev and Zh.B. Kurmanov, World Scientific, Singapore, 1995), p. 651-657; Preprint LPI No. 17 (1994).

[34] A.I. Lebedev and G.A. Sokol, Search for eta-nuclei, Preprint LPI No. 34 (1995).

[35] G.A. Sokol and V.A. Tryasuchev, A possible method of observing eta nuclei, Bull. Lebedev Phys.Inst., No.4 (1991) 21 [Kratk. Soobshch. Fiz. 4 (1991) 23 (in Russian)].

[36] M. Pfeiffer, J. Ahrens, J.R.M. Annand et al., Photoproduction of $\eta$-mesic ${ }^{3} H e$, Phys. Rev. Lett. 92 (2004) 252001; Ibid. 94 (2005) 049102.

[37] F. Pheron, J. Ahrens, J.R.M. Annand et al., Coherent photoproduction of $\eta$-mesons off ${ }^{3} \mathrm{He}-$ search for $\eta$-mesic nuclei, Phys. Lett. B 709 (2012) 21.

[38] S.V. Afanasiev, A.S. Artiomov, R.N. Bekmirzaev et al., Search results of $\eta$-mesic nuclei in the $d+\mathrm{C}$ reaction in JINR, Nucl. Phys. B (Proc. Suppl.) 209-210 (2011) 255.

[39] A. Budzanowski, A. Chatterjee, P. Hawranek et al., Search for $\eta$-mesic nuclei in a recoil-free transfer reaction, Phys. Rev. C 79 (2009) 012201(R).

[40] R.S. Hayano, S. Hirenzaki and A. Gillitzer, Formation of $\eta$-mesic nuclei using the recoilless $\left(d,{ }^{3} \mathrm{He}\right)$ reaction, Eur. Phys. J. A 6 (1999) 99.

[41] J. Kulpa and S. Wycech, The absorptive $\rho^{2}$ terms in the $\eta$ optical potential, Acta Phys. Pol. B 29 (1998) 3077.

[42] H. Calén, S. Carius, K. Fransson et al., The $p p \rightarrow p p \eta$ reaction near the kinematical threshold, Phys. Lett. B 366 (1996) 39.

[43] H. Calén, J. Dyring, K. Fransson et al., Measument of the quasifree $p+n \rightarrow d+\eta$ reaction near threshold, Phys. Rev. Lett. 79 (1997) 2642.

[44] H. Calén, J. Dyring, K. Fransson et al., Threshold structure of the quasifree $p+n \rightarrow d+\eta$ reaction, Phys. Rev. Lett. 80 (1998) 2069. 
[45] H. Calén, J. Dyring, K. Fransson et al., Measument of the quasifree pn $\rightarrow$ pn $\eta$ reaction, Phys. Rev. C 58 (1998) 2667.

[46] J. Smyrski, P. Wünster, J.T. Balewski et al., Near-threshold $\eta$ meson production in proton-proton collisions, Phys. Lett. B $\mathbf{4 7 4}$ (2000) 182.

[47] P. Moskal, R. Czyżykiewicz, H.-H. Adam et al., Near-threshold production of the $\eta$-meson via the quasifree pn $\rightarrow$ pn $\eta$ reaction, Phys. Rev. C 79 (2009) 015208.

[48] V. Baru, A.M. Gasparyan, J. Haidenbauer, C. Hanhart, A.E. Kudryavtsev and J. Speth, Production of $\eta$ mesons in nucleon-nucleon collisions, Phys. Rev. C 67 (2003) 024002.

[49] A.S. Iljinov, I.A. Pschenichnov, N. Bianchi et al., Extension of the intranuclear cascade model for photonuclear reactions at energies up to $10 \mathrm{GeV}$, Nucl. Phys. A 616 (1997) 575.

[50] A. Ignatov, O. Bartalini, V. Bellini et al., New experimental and simulated results on nuclear media effects in meson photoproduction off nuclei, Prog. Part. Nucl. Phys. 61 (2008) 253. 